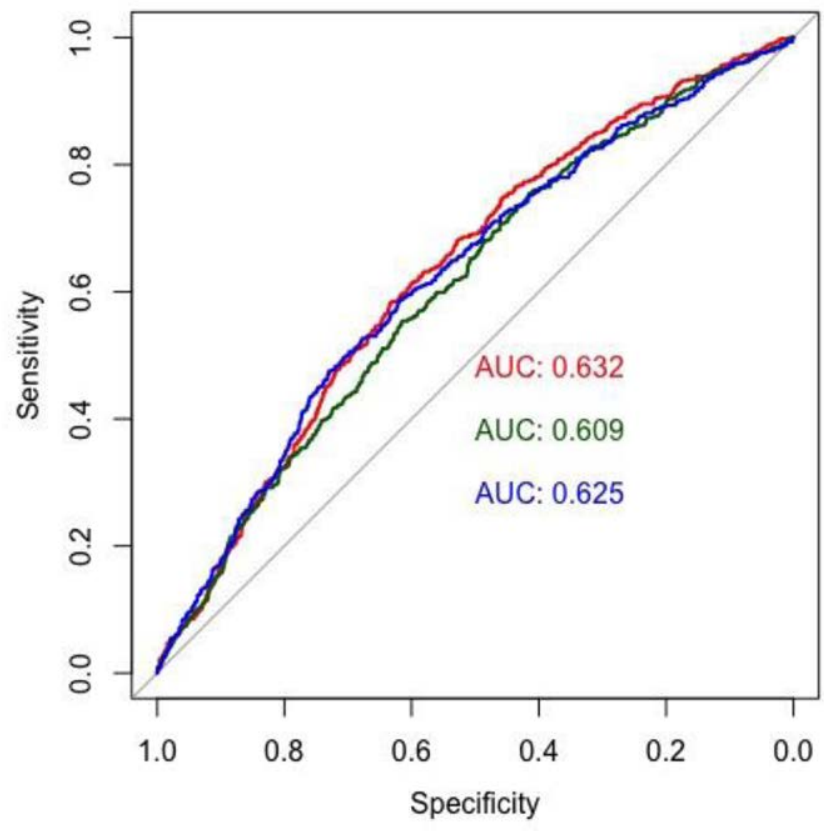

Figure 1. Receiver operating characteristic curves for CSA (red), CSMI (green) and SI (blue). AUC for CSA was 0.632, CSMI-0.609 and SI-0.625.

Conclusion: These data suggest that CSA, CSMI and SI help predict the fracture risk in patients with RA. HAL, D1, D2, D3, A, $\Theta$ and $Y$ do not predict risk of fracture. The CSA regression model was the strongest predictor of fracture. HSA measurements can therefore help predict risk of fracture in conjunction with other factors. Limitations of the study are that it was retrospective and only studied patients who had a DEXA scan.

REFERENCES:

[1] Xue A, Wu S, Jiang L, Feng A, Guo H, Zhao P. Bone fracture risk in patients with rheumatoid arthritis: A meta-analysis. Medicine. 2017; 96 (36): e6983. Available from: doi: 10.1097/MD.0000000000006983.

[2] Faulkner KG, Wacker WK, Barden HS, Simonelli C, Burke PK, Ragi S, Del Rio L. Femur strength index predicts hip fracture independent of bone density and hip axis length. Osteoporos Int. 2006;17(4):593-9. doi: 10.1007/ s00198-005-0019-4.

[3] Kaptoge S, Beck TJ, Reeve J, Stone KL, Hillier TA, Cauley JA, et al. Prediction of Incident Hip Fracture Risk by Femur Geometry Variables Measured by Hip Structural Analysis in the Study of Osteoporotic Fractures. Journal of Bone and Mineral Research. 2008; 23 (12): 1892-1904. Available from: doi: https://doi.org/10.1359/jbmr.080802.

Disclosure of Interests: None declared

DOI: 10.1136/annrheumdis-2021-eular.802

\section{POS0512 DIFFERENCES IN TREATMENT SATISFACTION, PATIENT PREFERENCES, AND TREATMENT PATTERNS BETWEEN EUROPEAN, SOUTH AMERICAN, AND JAPANESE PATIENTS WITH SUBOPTIMALLY CONTROLLED RHEUMATOID ARTHRITIS: A SUBGROUP ANALYSIS OF THE SENSE STUDY}

P. C. Taylor ${ }^{1}$, P. Sidiropoulos ${ }^{2}$, C. Ancuta ${ }^{3}$, I. Lagunes-Galindo ${ }^{4}$, M. Delavega ${ }^{5}$, U. Kalyoncu ${ }^{6}$, O. Nagy ${ }^{4}$, A. Kawakami ${ }^{7}{ }^{1}$ University of Oxford, Botnar Research Centre, Nuffield Department of Orthopaedics, Rheumatology and Musculoskeletal Sciences, Oxford, United Kingdom; ${ }^{2}$ University of Crete, Faculty of Medicine, Heraklion, Greece; "“"Grigore T. Popa” University of Medicine and Pharmacy, Department of Rheumatology, Iași, Romania; ${ }^{4} \mathrm{AbbVie}$, Inc,., North Chicago, United States of America; ${ }^{5} \mathrm{CEIM}$ Investigaciones Medicas,.., Buenos Aires, Argentina; ${ }^{6}$ Hacettepe University, Department of Internal Medicine, Division of Rheumatology, Ankara, Turkey; ${ }^{7}$ Nagasaki University Graduate School of Biomedical Sciences, Department of Immunology and Rheumatology, Nagasaki, Japan

Background: Despite the availability of advanced therapies, many patients with rheumatoid arthritis (RA) do not achieve their treatment goals. Understanding geographic influence on patient perspectives and physicians' attitudes toward treatment adjustments may inform region-specific strategies to improve outcomes in RA.

Objectives: To explore differences in treatment satisfaction, patient preferences, and treatment strategies between patients from Europe (EU), South America (SA), and Japan (JP) with suboptimal control of RA.

Methods: This is a subgroup analysis of SENSE, a non-interventional cross-sectional study in adults with RA who had moderate/high disease activity as measured by Disease Activity Score in 28 joints with erythrocyte sedimentation rate (DAS28[ESR]) >3.2, despite disease-modifying antirheumatic drug (DMARD) treatment. Patient satisfaction and preferences, treatment adherence, patient-reported outcomes, and physicians' plans for DMARD switch were assessed as previously described. ${ }^{1}$ Predictors of good treatment satisfaction and physician's decision not to switch DMARDs were identified using multiple logistic regression analyses.

Results: Of 1234, 272, and 118 patients enrolled from EU, SA, and JP, respectively, $13.9 \%, 15.4 \%$, and $5.9 \%$ reported good treatment satisfaction. Irrespective of region, patients reported impaired quality of life and good treatment adherence, and around one-third of patients received targeted synthetic (ts) or biologic (b) DMARDs. Among patients treated with ts/bDMARDs, monotherapy was most common in SA $(45.3 \%)$, followed by EU $(26.3 \%)$ and JP (18.8\%), consistent with a greater acceptance of combination therapy in JP. More than $80 \%$ of JP patients preferred oral treatments versus $<60 \%$ of EU/SA patients. DMARD switches were planned in $51.8 \%$ (EU), $57.4 \%$ (SA), and $38.1 \%$ (JP) of patients, most commonly to a tumor necrosis factor inhibitor. Predictors for good treatment satisfaction included treatment with ts/bDMARDs and the presence of psychiatric disorders in EU and SA; however, current disease activity was not a common predictor (Table 1). Reluctance to switch treatments was predicted by lower disease activity assessed by DAS28(ESR) (all regions) and current treatment with ts/bDMARDs (EU/SA).

Table 1. Predictors for good treatment satisfaction and no treatment switch planned

\begin{tabular}{cccc}
\hline OR $(95 \% \mathrm{Cl}) \quad \begin{array}{c}\text { Europe } \\
(n=1234)\end{array}$ & $\begin{array}{c}\text { South America } \\
(n=272)\end{array}$ & $\begin{array}{c}\text { Japan } \\
(n=118)\end{array}$
\end{tabular}

Good treatment satisfaction (TSQM global treatment satisfaction $\geq 80$ )

Current treatment with ts/bDMARDs $3.8(2.7,5.4)^{\star \star \star \star *} \quad 4.9(2.2,10.8)^{\star \star \star * *}$

Psychiatric disorders

Number of comorbidities

Worst joint pain

SF-36 MCS

SF-36 PCS

Work Productivity and Activity

Impairment-Rheumatoid Arthritis:

Total activity impairment

DAS28(ESR) $>5.1$

Female

No treatment switch planned

Current treatment with ts/bDMARDs

Number of comorbidities

Age

TSQM effectiveness subscore

Number of concomitant medications

DAS28(ESR)

\begin{tabular}{|c|c|c|}
\hline $3.8(2.7,5.4)^{\star \star \star \star}$ & $4.9(2.2,10.8)^{\star \star \star \star}$ & - \\
\hline $2.4(1.3,4.6)^{\star \star}$ & $3.1(1.2,8.4)^{\star}$ & - \\
\hline- & - & $2.3(1.2,4.3)^{*}$ \\
\hline- & $1.3(1.1,1.5)^{\star *}$ & \\
\hline $1.0(1.0,1.1)^{\star \star \star \star}$ & - & $1.3(1.1,1.5)^{\star}$ \\
\hline $1.1(1.0,1.1)^{\star \star \star \star}$ & - & - \\
\hline- & $1.0(1.0,1.0)^{\star \star \star}$ & - \\
\hline - & $0.3(0.1,0.7)^{\star \star}$ & - \\
\hline - & - & $0.1(0.0,0.9)$ \\
\hline $3.9(3.0,5.2)^{\star \star \star \star}$ & $2.4(1.4,4.2)^{\star \star}$ & - \\
\hline $1.2(1.1,1.4)^{\star \star \star \star}$ & - & - \\
\hline- & - & $1.0(1.0,1.1)^{*}$ \\
\hline $1.0(1.0,1.0)^{\star \star \star}$ & $1.0(1.0,1.0)^{\star \star *}$ & - \\
\hline $0.9(0.8,1.0)^{\star \star}$ & - & - \\
\hline $.5(0.5,0.6)^{\star \star \star \star}$ & $0.6(0.5,0.8)^{\star \star \star}$ & $0.6(0.4,0.9)$ \\
\hline
\end{tabular}

$\mathrm{X}^{2}$ test: ${ }^{* * * *} \mathrm{p}<0.0001,{ }^{* * *} \mathrm{p}<0.001,{ }^{* *} \mathrm{p}<0.01,{ }^{*} \mathrm{p}<0.05 \mathrm{Cl}$, confidence interval; OR, odds ratio SF-36 M/PCS, 36-Item Short-Form Survey mental/physical component summary; TSQM, Treatment Satisfaction Questionnaire for Medication

Conclusion: In patients with moderate to high RA disease activity, current disease control was a common determinant of treatment switches. Predictors for good treatment satisfaction revealed region-specific patient attitudes to treatment acceptance despite poor disease control.

\section{REFERENCES:}

[1] Taylor PC, et al. Ann Rheum Dis 2020;79:996-7

Acknowledgements: AbbVie funded this study; contributed to its design; participated in data collection, analysis, and interpretation of the data; and participated in the writing, review, and approval of this abstract. No honoraria or payments were made for authorship. Medical writing support was provided by Hilary Wong, $\mathrm{PhD}$, of 2 the Nth (Cheshire, UK), and was funded by AbbVie.

Disclosure of Interests: Peter C. Taylor Speakers bureau: AbbVie, Biogen, Bristol-Myers Squibb, Celgene, Eli Lilly, Fresenius, Galapagos, Gilead, GSK, Janssen, Nordic Pharma, Pfizer, Roche, Sanofi, and UCB., Consultant of: AbbVie, Biogen, Bristol-Myers Squibb, Celgene, Eli Lilly, Fresenius, Galapagos, Gilead, GSK, Janssen, Nordic Pharma, Pfizer, Roche, Sanofi, and UCB., Grant/research support from: AbbVie, Biogen, Bristol-Myers Squibb, Celgene, Eli Lilly, Fresenius, Galapagos, Gilead, GSK, Janssen, Nordic Pharma, Pfizer, Roche, Sanofi, and UCB., Prodromos Sidiropoulos Speakers bureau: AbbVie, Amgen, MSD, Novar tis, Pfizer, Roche, and UCB., Consultant of: AbbVie, Amgen, MSD, Novartis, Pfizer, Roche, and UCB., Grant/research support from: AbbVie, Amgen, MSD 
Novartis, Pfizer, Roche, and UCB., CODRINA ANCUTA Speakers bureau: AbbVie, Eli Lilly, Ewopharma, MSD, Novartis, Pfizer, Roche, and UCB., Consultant of: AbbVie, Eli Lilly, Ewopharma, MSD, Novartis, Pfizer, Roche, and UCB. Ivan Lagunes-Galindo Employee of: AbbVie employee and may own stocks or options, Maria DeLaVega: None declared, Umut Kalyoncu Speakers bureau: AbbVie, Amgen, Eli Lilly, Janssen, Novartis, Pfizer, Roche, and UCB., Consultant of: AbbVie, Amgen, Eli Lilly, Janssen, Novartis, Pfizer, Roche, and UCB., Orsolya Nagy Employee of: AbbVie employee and may own stocks or options Atsushi Kawakami Speakers bureau: AbbVie, Actelion, Asahi Kasei, Astellas Boehringer Ingelheim, Celltrion, Chugai, Daiichi Sankyo, Eisai, Eli Lilly, GSK, Janssen, Kowa, MedPeer, Mitsubishi Tanabe, Novartis, ONO, Pfizer, Taisho, and Takeda, Grant/research support from: AbbVie, Actelion, Asahi Kasei, Astellas, AYUMI, Boehringer Ingelheim, Bristol-Myers Squibb, Celltrion, Chugai, Daiichi Sankyo, Eisai, Eli Lilly, Kyowa Hakko Kirin, MSD, Neopharma, Novartis, ONO, Sanofi, Taisho, Takeda Science Foundation, and Teijin. DOI: 10.1136/annrheumdis-2021-eular.842

\section{POS0513 ABATACEPT IN MONOTHERAPY VERSUS COMBINED IN INTERSTITIAL LUNG DISEASE OF RHEUMATOID ARTHRITIS. MULTICENTER STUDY OF 263 CAUCASIAN PATIENTS}

C. Fernández-Díaz ${ }^{1}$, B. Atienza-Mateo ${ }^{1}$, S. Castañeda ${ }^{2}$, R. Melero ${ }^{3}$, F. OrtizSanjuán $^{4}$, I. Casafont-Solé ${ }^{5}$, J. Loricera ${ }^{1}$, S. C. Rodriguez-García ${ }^{6}$, I. FerrazAmaro $^{7}$, M. A. González-Gay ${ }^{1}$, R. Blanco on behalf of Spanish Collaborative Group of Interstitial Lung Disease Associated to Rheumatoid Arthritis Members of the Spanish Collaborative Group of Interstitial Lung Disease Associated to Rheumatoid Arthritis: Clara Aguilera-Cros (H.U. Virgen del Rocío, Sevilla), Ignacio Villa (H. Sierrallana, Torrelavega), Enrique Raya-Alvarez (H.U. San Cecilio, Granada), Clara Ojeda-García (H.U. Virgen Macarena, Sevilla), María G. Bonilla Hernán (H.U. La Paz, Madrid), Ana M. López-Robles (H.U. Vall d'Hebron, Barcelona), Luis Arboleya-Rodríguez (H.U. Central de Asturias, Asturias), Javier Narváez García (H.U. Bellvitge, Barcelona), Evelin C. Cervantes Pérez (H.U. de Santiago, Santiago de Compostela, A Coruña), OlgaMaiz-Alonso (H.U. Donostia, Gipuzkoa), María N. Alvarez-Rivas (H.U. Luca Augusti, Lugo), Iván Cabezas-Rodríguez (H.U. Río Hortega, Valladolid), Eva Salgado-Pérez (C.H.U. Ourense, Ourense), Cristina Hidalgo-Calleja (H.U. de Salamanca, Salamanca), Sabela Fernández-Aguado (H.U. Cabueñes, Asturias), Jesús C. Fernández-López (C.H.U. A Coruña), Alejandro Olivé, Samantha Rodríguez-Muguruza (H.U. GermansTrias i Pujol, Barcelona), Raquel Almodóvar-González (H.U. Fundación Alcorcón, Madrid), Carmen Carrasco-Cubero (C.H.U. Infanta Cristina, Badajoz), Antonio Juan-Mas (H. Son Llàtzer, Palma de Mallorca), Raúl Castellanos-Moreira (H.U. Clinic. Barcelona), Iñigo Hernández Rodríguez (C.H.U. de Vigo, Vigo), Neus QuillisMarti (H. Vinalopo Elche), José A. Bernal-Vidal (H. Marina Baixa, Villajoyosa), Angel García-Aparicio (H. Virgen de la salud, Toledo), Sonia Castro-Oreiro (H.U. Joan XXIII, Tarragona), Julia Fernández-Melón (H. Son Espases, Palma de Mallorca), Paloma Vela Casasempere(H. U. Alicante, Alicante), María C. Fito, Carmen González-Montagut (C.U. Navarra, Navarra), Manuel Rodríguez-Gómez (C.H.U. Ourense, Ourense), Trinidad Pérez-Sandoval, Miriam Retuerto-Guerrero (H.U. León), Deseada Palma-Sánchez (H. Rafael Mendez, Lorca), José L. Andreu (H. U. Puerta del Hierro), Patricia CarreiraDelgado (H. 12 de Octubre, Madrid), Lorena Expósito-Pérez (H.U. de Canarias, Tenerife), Juan RD Jiménez- de Aberásturi, Ana Ruibal-Escribano (H.U. Txagorritxu, Araba), Ana Urruticoechea-Arana (H. Can Misses, Ibiza), Rosa Expósito-Molinero (H. Laredo, Laredo), Rubén López Sánchez (H. Negrín Las Palmas), Manuel J.Moreno-Ramos (H. Virgen de la Arrixaca, Murcia), Isabel Serrano-García (H. Puerta del Mar Cadiz), Blanca García-Magallón (H. San Jorge Huesca), José María Andreu Ubero (H. Virgen de las Nieves Granada), Natalia Mena-Vázquez (H.R.U Málaga), Iván Castellvi-Barranco (H.U. De la Santa Creu i Sant Pau, Barcelona), Carmen González-Montagut (H. U. Valladolid, Valladolid), Juan Blanco-Madrigal (H. Basurto, Bilbao), Pilar Morales-Garrido (H.U. San Cecilio, Granada), Cilia Peralta-Ginés (H. Lozano Blesa, Zaragoza), Mireia López-Corbeto (H.U. Vall d’Hebron, Barcelona), Sergio Ordóñez-Palau (H. Lleida, Lleida), Andrea García-Valle (H. U Palencia), Susana Romero-Yuste (H.U. Pontevedra, Pontevedra).. ${ }^{1}$ Marqués de Valdecilla University Hospital, Rheumatology, Santander, Spain; ${ }^{2}$ Hospital de La Princesa, Rheumatology, Madrid, Spain; ${ }^{3}$ Complejo Hospitalario Universitario de VIgo, Rheumatology, Vigo, Spain; ${ }^{4}$ Hospital La Fe, Rheumatology, Valencia, Spain; ${ }^{5}$ Hospital Germans Trias i Pujol, Rheumatology, Badalona, Spain; ${ }^{6}$ Hospital Clínic, Rheumatology, Barcelona, Spain: ${ }^{7}$ Hospital Universitario de Canarias, Rheumatology, San Cristóbal de La Laguna, Spain
Background: Interstitial lung disease (ILD) is a severe complication of RA. Abatacept (ABA) have demonstrated efficacy in RA-ILD [1,2], although combined treatment with MTX or others DMARDs remain controversial.

Objectives: To assess the efficacy and safety of $A B A$ in monotherapy ( $\left.A B A_{M O N O}\right)$ versus combined-ABA, ABA plus MTX(ABA $\left.A_{M T X}\right)$ or ABA plus other non-MTX DMARDs (ABA NON-MTX $_{\text {T }}$ ), in RA-ILD.

Methods: Observational multicenter study of RA-ILD caucasian patients treated

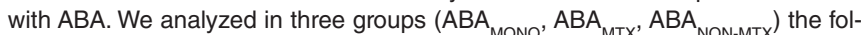
lowing outcomes: a) Dyspnea, b) FVC and DLCO, c) HRCT, d) DAS28-ESR, e) corticosteroid-sparing effect. Differences between basal and final follow-up were evaluated. Multivariable linear regression was used between the three groups. Results: We studied 263 RA-ILD patients (mean age 64.6 \pm 10 years) [ABA $(n=111), A^{-1 A_{M T X}}(n=46)$ and $\left.A_{B A} A_{\text {NON-MTX }}(n=106)\right]$. At baseline, $A B A_{\text {MONO }}$ patients were older ( $67 \pm 10$ years) and took higher prednisone dose (10 [IQR 5-15] mg/ day). There was no statistically significant differences in sex, seropositivity, ILD patterns, FVC, DLCO or disease duration. In all groups, most patients experienced stabilization or improvement in FVC, DLCO, dyspnea, HRCT as well as improvement in DAS28-ESR. A statistically significant difference between basal and final follow-up was only found in corticosteroid-sparing effect in $\mathrm{ABA}_{\mathrm{MTX}}$ or $\mathrm{ABA}_{\mathrm{NON}-\mathrm{MTX}}$ (Figure 1). However, in the multivariable analysis, there were no differences in any outcome between the three groups(Table 1).
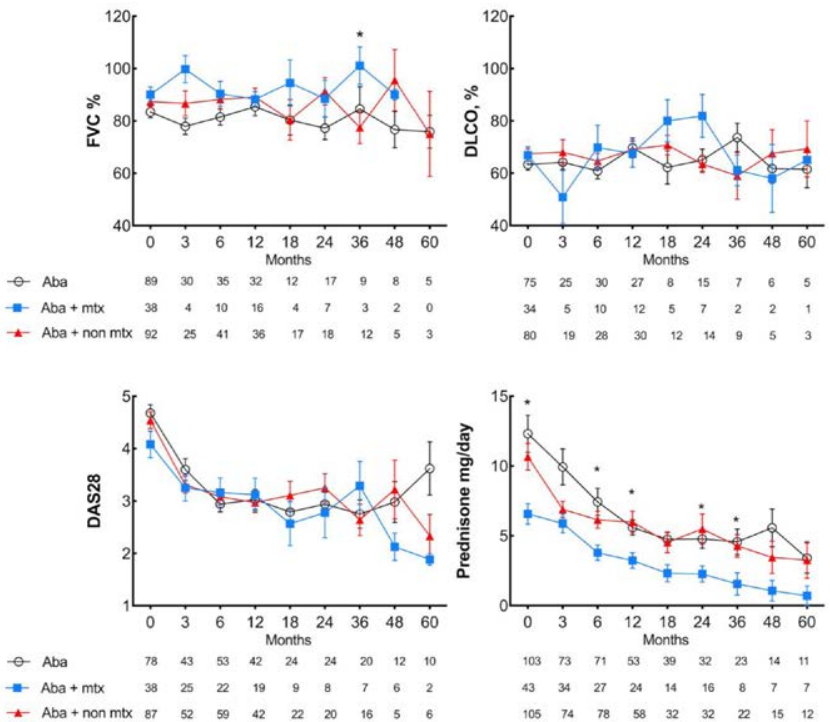

$-A b a+m t x$

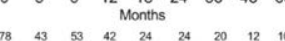

$\begin{array}{lllllll}105 & 74 & 78 & 58 & 32\end{array}$

Figure 1.

Conclusion: In caucasian individuals with RA-ILD, ABA ${ }_{\mathrm{MONO}}$ or $\mathrm{ABA}_{\mathrm{MTX}}$ or ABANON-MTX seems to be equally effective and safe. However, a corticosteroid-sparing effect is only observed in combined-ABA

\section{REFERENCES:}

[1] Fernández-Díaz C, et al. Abatacept in patients with rheumatoid arthritis and interstitial lung disease: A national multicenter study of 63 patients. Semin Arthritis Rheum. 2018 Aug;48(1):22-27. doi: 10.1016/j.semarthrit.2017.12.012.

[2] Fernández-Díaz C, et al. Abatacept in interstitial lung disease associated with rheumatoid arthritis: national multicenter study of 263 patients. Rheumatology (Oxford). 2020 Dec 1;59(12):3906-3916. doi: 10.1093/rheumatology/ keaa621.

Acknowledgements: Spanish Collaborative Group of Interstitial Lung Disease Associated to Rheumatoid Arthritis

Disclosure of Interests: Carlos Fernández-Díaz Speakers bureau: Roche, bristol myers squibb, Belén Atienza-Mateo: None declared, Santos Castañeda: None declared, Rafael Melero: None declared, Francisco Ortiz-Sanjuán: None declared, Ivette Casafont-Solé: None declared, J. Loricera: None declared, Sebastián C Rodriguez-García: None declared, Iván Ferraz-Amaro: None declared, Miguel A González-Gay: None declared, Ricardo Blanco Speakers bureau: bristol myers squibb

DOI: 10.1136/annrheumdis-2021-eular.892 